The earliest operation, undertaken under most unfavorable conditions, though it did not succeed, was encouraging. I think I may safely assert that the results, on the whole, would have been better if every case had been explored within twenty-four hours of the onset of pain. We should probably have saved all the lives and should have avoided much unnecessary risk and suffering.

TREATMENT OF CHRONIC ENDOME'TRITIS BY CURE'I'ING ANI) (GAUZE DRAINAGE : IVITII A SYNOPSIS OF 'IWEN'TY-SEVEN CASES.

BY WALTER I. BURLAGK, A.M. M.b.

To Dr. William M. Polk, of New York, is due in a large measure the credit of having developed and perfected a method of treating endometritis that is rapid in its performance and eflicacious in its results.

The method consists of divulsion, curetting and thorough irrigation of the uterus and then packing it lightly with an antiseptic wicking of gauze, the gauze being allowed to remain in place for several days.

Although gauze has been ernployed as a uterine packing time out of mind, it had not been used in just this manner until Dr. Polk so used it, and called the attention of the profession to the matter in a paper read before the Practitioner's' Society of New York in the spring of 1888 . In this paper he spoke brietly of the unsatisfactory results obtained by the usual methods of treatment of endometritis. $\Lambda$ consideration of the conditions present in endometritis, he said, should lead to the institution of a more rational therapy. In this affection there is an inflamed and suppurating surface lining a nearly closed cavity, and tho conditions are almost exactly comparable to those present in an abscess with an opening of insufficient size for tho free escape of pus. The discharge is retained in the cavity bathing the already inflamed surfaces, keeping up the irritation, and preventing thereby any attempts on the part of nature to effect a cure. In the case of an abscess-cavity with a small opening the plain indications are to enlarge the opening aud drain away all the irritating discharges as rapidly as they are formed, and a similar wode of procedure should be equally effective in the analogous condition found within the uterus in endometritis.

Again, in the same year (1888) he read a paper before the American Gynacological Society on this subject, and referred to nine cases he had treated with success. Last December, in a third paper, before the New York Academy of Medicine, Dr. Polk advocated his plan of treatment in cases of endometritis associated with salpingitis or other periuterine inflammation, opposing the ground taken by most writers of textbooks, that where there is periuterine inflammation, the cavity of the uterus should not be invaded. He reported forty cases.

There is no doubt that treating the uterus in this way is a radical procedure; it is, however, founded on solid surgical principles, on cleanliness and drainage. The idea that pelvic inflammation following the passage of the sound was due exclusively to violence is giving way to the belief that the inflummatory trouble was caused largely by lack of asepsis, as to the sound, the vagina or both.

' Leml before the Boston Society for Medioal Observation, Docomber $\mathrm{b}, 1892$.
In case of salpingitis, ovaritis, retroversion and pathological anteflexion, it has been my experience that by correcting the endometritis in the method about to be described the symptoms of these affections have been ameliorated. Curetting and drainage should accompany the Alexander-Adams operation for retroversion, for the reason that the replacing of the uterus is not alone sufficient to overcome the deep-seated inflammatory condition of the endometrium that is found in retroversion of long standing: 80 in many cases of old lacerated cervix, the accompanying endometritis persists for years if not treated at the time of repairing the cervix. The same is true in some cases of coliotomy for the removal of pus tubes. We have all seen bothersome hemorrhagic endometritis causing annoyance a long time after the tubes and ovaries were out. It certainly seems to be a more rational plan to treat all the disease at once, rather than a part of it.

Curetting and gauze drainage confine a patient to the house for from ten days to threo weeks. 'The average stay in the hospital of l'olk's forty cases was eighteen days. I have not looked up the statistics of mine, but should say the average was a little lower. Most of the older methods of treatment, besides not being as thorough, took months of painful applications, and then the results were far from satisfactory. One especial advantage of this method is that the physician has the immense benefit of an ether examination, a procedure of inestimable value in the diagnosis of pelvic affections in women.

Chronic endometritis will be treated of in this paper from a clinical point of view. The different anatomical varieties enumerated by Pozzi are, - interstitial, glandular, polypoid, and the lesions of the cervix, and inflammation of the ovules of Naboth, granulations and folliculitis. A positive diagnosis of many of these without a microscopic examination of the scrapings is impossible. I have classified the cases as hamorrhagic, painful, catarrhal and purulent. Where there has been good reason to believe from the history that a purulent discharge is infected with the gonococcus, it has been called gonorrlocal. I have not demonstrated microscopically the presence of the gonococcus in any of the cases reported to-night. By endometritis is meant an inflammation of tho endometritium involving in most instances both cervix and body. It is a mistake, I believe, to consider inflammation of the endometrium of either the cervix or tho body as a circumscribed lesion. 'Tho disease is apt to be more pronounced in one than the other, but treating one to the exclusion of the other gives unsatisfactory results.

The symptoms of endometritis, broudly, are pelvic pain, leucorrhca, irregular catamenia, dysmenorrhou, metromhagia or scauty menses, bladder and rectal symptoms, and reflex neuroses. Common symptoms are dyspepsia, neuralgia, constipation, sleeplessness and luck of energy and strength.

The physical signs are alteration in consistency and volume of the cervix, sensitiveness to light pressure bimanually of the cervix, body or both; the presence of erosions and dilated Nabothian follicles; a discharge from the os of clear transparent white-of-egg mucus, or opaque tenacious mucus, pus or blood, or a combination of any of these. Passage of the probe or sound shows increased uterine depth and is attended by more or less pain.

'The hxmorrhagic form effects the boly more than the cervix. The diagnosis is estublished by almost 
continual losses of blood through many months, the flow being unattended by pain as a rule, though in this variety there is likely to be constant lumbar pain ; by finding on physical examination a uterine interior that has a boggy feel to the examining probe or sound, and the probe on withdrawal being followed by a flow of blood. In the painful form constant pain in the pelvis, and its consequent weakness, are the chief features. It has an insidious course; respites and exacerbations are to be expected. It seems to be the result of an infection that has developed slowly, and not the sequel to an acute attack. 'The cervix is hard, even sclerosed ; in many cases it is abnormally long and the canal very tight, admitting a probe with difficulty; the canal is very sensitive; the dischargo is either clear, transparent, white-of-egg, or viscid, opaque mucus. 'The catarrhal and purulent forms are characterized by abuudant cervical discharge and by erosions of the cervix. The disease in this form is more pronounced in the cervix. There are many reflex nervous symptoms.

It is not my purpose to go into the symptoms nor diagnosis of chronic endometritis at length. Enough has been said to show on what features my diagnoses are based. We will proceed to the consideration of the operative treatment.

The operation of curetting the uterus is the one operative gynecological procedure that is done most often by the general practitioner. There are so many different methods, many of them faulty either in lack of asepsis or thoroughuess, that it seems worth while to de. scribe in detail a method that if carried out in all its minutia is accompanied by uniformly favorable results.

An anasthetic is a necessity in cases of chronic endometritis. Curetting - following abortion, and in certain fibroids where the canal is patulous and the uterus non-sensitive - may be done with cocaine or even without that. I have used a two-per-cent. solution of cocaine, injected with a hypodermic syringe in two or three places in the walls of the cervical canal. This does away with most of the seusitiveness, which, as a rule, is situated in the cervix and about the internal os rather than at the fundus.

Now as to curetting for chrouic endometritis. The instruments needed are: two divulsors, a small light one with narrow blades bent at a good angle, and a large and strong one, with corrugations to prevent slipping: oftentimes Hanks hard-rubber dilators will be sutlicient; a sound; dressing-forceps; bivalve speculum (Graves); American bullet-forceps; cervical speculum; tampon screw, or forked pusher; pair of ordinary scissors and curette. A word as to the curette. I have had considerable experience with the blunt wire loop and with Emmet's curette forceps, and they are neither satisfactory; the dull wire because being dull, it does not scrape well (the only oflice of a curette), and the curette forceps (a valuable instrument for pinching off mucous polypi), because with it you cannot go over the entire endometrium thoroughly and remove all the disease. The curette I like best is a modification of Sims's sharp curette, modified in being less sharp, the loop staller, and the staff slender, flexible and elastic, so that the scraping is done with a non-rigid instrument. 'Thus you do away with the danger of going too deeply and of perforation. The harm to be derived from scraping away the endometrium, if done with reasonable gentleness, is to my mind chimerical. The trouble that resulted as it was done in the days of Sims, looking at the question from our stand-point to.day, was due less to the removal of the tissue than to lack of asepsis. If the endometrium is diseased, it is a rational process to remove it, especially as we have every reason to believe it is readily reformed. $A_{8}$ to the divulsors: the small one is for tight canals, met most of ten in anteflexed uteri ; the large one (Warthen's I prefer), is to complete the stretching. One divulsor is not enough. The forked pusher is a slender steel rod with metal handle, the rod being tipped with a shallow $U$, the prongs of which engage the gauze and carry it in to the uterus. The cervical speculum is the only other instrument that needs special mention. In tight virgin cervices, particularly in the long anteflexed variety, it is next to impossible to wash out the uterine cavity before and after curetting with any ordinary irrigator because the cervix shuts down tightly on the tube preventing a return flow; hence the danger of forcing irrigating fluid and débris from the endometrium through the Fallopian tubes into the peritoneal cavity. This has been the bugaboo of irrigation in the past. Most intra-uterine irrigators and double-current apparatuses are complicated and hard to keep clean, and the open-

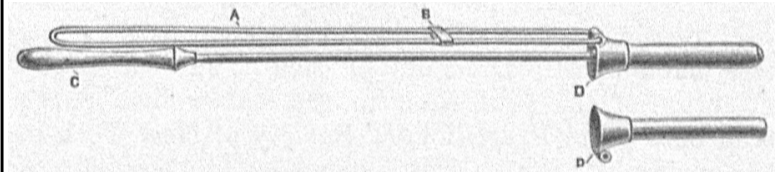

ings for the return flow are apt to become clogged by clots and tissue while in use. In placing the gauze after the irrigation, the mucous membrane of the cervical canal having been rendered adhesive by the corrosive, it is found to be diflicult to get the gauze through the cervix up to the fundus; but with the cervical canal lined with a sheath of polished metal, placing the gauze becomes an easy matter. The instrument I use is one I modified from Polk's original pattern, now no longer wanufactured. It has two cylinders, as you see, with a detachable handle, and two obturators, one fitting each cylinder, and tixed on either end of a flexiblo staff. The larger cylinder, No. 28, French, is for use with the patient aniesthetized, and the smaller one, No. 25, French, is for subsequent treatments, should they prove necessary. In the cut, $D$ D are the cylinders, A the detachable wire handle with its sliding clamp B, and $\mathrm{c}$ the obturator that is not in the cylinder. All the parts are metal, nickel plated. The instrument is made by Codwan \& Shurtleff of this city.

This completes the list of instruments. Other necessary apparatus consists of a basin of corrosive (13000 ), and a half-dozen balls of absorbent cotton about an inch in diameter, to be used with the dressingforceps for sponging. They are us useful as sponges and sponge-holders and far less trouble and cleaner, as a ball of cotton is used but once and thrown away. For irrigation, an ordinary douche-pail or bag lung on a peg or the gas-fixture near by and filled with warm corrosive (1-3000), a piece of glass tubing, six to eight inches long, and of a diameter of not over three-sixteenths of an inch, should be fitted to the end of the rubber tube from the douche-bag. Prepare two pieces of plain gauze, each double thickuess, one-half to three-quarters of an inch wide and three feet long. Sterilize them. Having cleansed the hands, impregnate one strip with iodoform or dermatol by rubbing it in a clean basin with the powder; place the other plain strip with it and have the basin within reach. 
While the patient is being prepared for the ether, the operator prepares his hands and forearms by washing in soap and hot water with a nail-brush for five minutes by the clock, changing the wash water at least five times. 'Then he stains them brown with a saturated solution of permanganate of potash, and completes the preparation by dissolving the color in a saturated solution of oxalic acid and rinsing in warm water.

'The patient's bladder and rectum having been emptied she is ancsthetized and placed on a table on her back in the lithotomy position, with her buttocks at the edge, and resting on a Kelly apron or obstetric pad, the flap of which is gathered into a pail on the floor. A Clover or Ot leg.holder is a handy thing to have as it does away with the need of an assistant to hold each leg. With this appliance, and one assistant to give the ether, it is possible to do a very thorough, clean and speedy operation.

'The instruments, previously sterilized by steaming in an Arnold sterilizer for half an hour or boiling in a one-per-cent. solution of washing soda for ten minutes, are laid on a sterilized towel on a table within easy reach of the operator's right hand, as he stands facing the patient at the foot of the table.

'The first step in the operation is a thorough bimanual examination. 'This should never be omitted, for, no matter how good an examination may have been had without ether, it is always possible that some important fact as to the condition of the uterus or the adnexa may be brought out when the abdominal walls are thoroughly relaxed by the anasthetic. In certain stout patients and in those with rigid abdominal walls, a satisfactory examination can be obtained only in this way. The biuanual examination is to be made before scrubbing up the field of operation, for the reason that after the vagina has been treated with corrosive it becomes rough and sticky, and thus interferes with the tactile sense. Next scrub the external genitals and buttocks with a nail-brush and soap and water. Hooking back the perineum with the left forefinger, introduce a piece of soap the size of a prune in the vagina and pour in a little water with it. With a rotary movement of the forefinger and middle finger of the left hand in the vagina wash it thoroughly. Hooking back the perineum again, pour water into the vagina from a basin until it runs out free from soap. Irrigate the vagina and exterual genitals with corrosive from the basin in the same way. We have now a clean field for operation. Insert the bivalve speculum in the vagina and seize the anterior lip of the cervix with the bullet-forceps. 'The forceps make two small holes only and serve to steady the uterus during the entire operation, and are preferable to the tenaculum, which is apt to tear out again and agnin.

Pass the sound to determine the patency, direction and depth of the uterine canal, and also the condition of the interior of the uterus. A pedunculated fibroid or abundant fungosities may be diagnosticated and located by this means.

Divulse the cervix. For this purpose, all the time holding it immovable by means of the bullet-forceps held in the left hand, pass the closed blades of the small dilator into the canal and through tho internal 08. Dilate slowly. Further dilatation is accomplished with the large Warthen dilatur. A moderate degree of stretching is sufficient. 'I'en minutes or more should be devoted to this part of the operation, as with quick divulsion, especially in a cervix having inelastic tissu $e 8$ it is an easy matter to tear through into the broad ligament. In this connection let me say, Don't approximate the handles of the large dilator by means of the thumb-8crew on them. You cannot estimate the force you are using. Bring the handles toward each other by the grasp of the hand until you think you have put the tissues sufficiently on the stretch, and then turn the screw to hold the handles as they are until the resistance is overcome. In most cases after dilatation you should be able to pass a No. 20 Hanks dilator with ease. Having removed the dilator, introduce the large cervical speculum. Irrigate for a moment the interior of the uterus by inserting the small glass-tube through the speculum to the fundus, lirst being careful that the water is running warm. liemove the speculum and introduce the curette to the fundus, and scrape in turn and thoroughly the anterior and posterior walls and the cornua, devoting the most attention to the seat of the lungosities, if present, and to the region of the internal os. It is here that the disease seems mostly to lurk, and a tight internal os is the chief obstacle to drainage. It is well to curette until all soft tigsue has been removed. When this has been accomplished the curette makes a characteristic grating sound on the uterino wall. Next, tho cervical canal should bo attended to, the walls receiving a good scraping. Pass the uterine speculum again and irrigate the cavity thoroughly like any abscess-cavity aud until the wash-water runs out clear. Leaving the speculum in place, take your strip of plain gauze from the basin and engage one end in the tip of the tampon screw or pusher, whichever instrument you happen to have. Carry the gauze to the fundus to one cornu, disengage the screw and withdraw it until its end is at the region of the inner extremity of the uterine speculum. 'Take a fresh hold of the gauze there and carry it to the fundus and to the other cornu, and so on until the cavity of the uterus is full. Pulling on the free end, withdraw all the gauze. 'The uterine cavity is now dry and free from corrosive. 'The next move is to pack it with iodoform or dermatol gauze, and the packing is carried on as just described, care being taken to pack from inside the uterus and not from outside the speculum. In the latter event you will find that the speculum has been filled instead of the cavity of the uterus. In packing, the gauze should be carried first into one horn und then into the other, and so on until the cavity is lightly tilled, remembering that the shape of the interior is similar to an isosceles triangle of solid dimensions flattened from front to back with its base at the fundus and its apex at the internal os. A forcible packing is likely to excito subsequent uterine coutractions. Now holding the gauze in place with the tampon screw remove the uterme speculum. Cut off with the sterilized scissors any excess of gauze, leaving about un iuch projecting from the os. Having removed the bullet-forceps, wiped out the vagina with a piece of absorbent cotton and taken out the bivalve speculum the operation is finished. It is my custom to place a suppository of ayueous extract of opium (one grain) and oxtract of belladomna (one-half grain) in the rectum before putting the pationt to bed. The entire operation should occupy about twenty minutes.

As to the after-treatment. The patient is kept in bed for from three to four days to a week; she is assisted by the nurse to sit up to pass urine, the use of the catheter being avoided as far as possiblo; after 
passing water the vulva is sprayed off with warm water. A vaginal douche of four quarts of hot corrosive (1 to 5000 , temperature $110^{\circ}$ to $115^{\circ}$ ) is given every night and morning while the patient is under treatment. The bowels are moved on the third day. Occasionally there is a rise of temperature on the second or third day. This generally means defective drainage. Any temperature above $101^{\circ}$ after the first night means danger. Now and then one meets with an irritable and intolerant uterus, where the patient suffers from cramp pain in the abdomen on the second or third day. The pains are sometimes relieved by the spontaneous expulsion of the gauze, at others they pass away in an hour or two. If not, the indication is, as in the case of a temperature above $101^{\circ}$, for the removal of the gauze. In removing the gauze it is important to observe the same antiseptic precautions with reference to the hands and instruments as with an operation. By passing the left forefinger to the external os and sliding the tampon screw along it, it is an easy matter to take out the gauze without disturbing the patient in bed.

$\Lambda t$ the end of a week the patient is placed on the table is the Sims position, a small Sims speculum passed, and the gauze removed. Should there still be present much sensitiveness of the cervix or fundus, accompanied by an unhealthy discharge, and this condition I have found mostly in cases of long-standing anteflexion, fix the cervix with tenaculum or bulletforceps and introduce the uterine speculum, using the small cylinder, No. 25 , and irrigate the cavity of the uterus with warm corrosive (1 to 3000). Dry it out and repack with antiseptic gauze. It has been my oxperience that the cervical canal remains well open when gauze has been introduced. Generally the small specu. lum will pass with only a momentary pain. In certain cases where the gauze has been expelled early, it may be necessary to pass Hanks dilators up to No. 16, when it will be found that the speculum enters easily. Putting in the gauze through the speculum is rarely accompanied by pain, due to the fact that the cervix, the sensitive portion, is protected by the speculum from contact with the gauze.

One or two packings, a week apart, are generally sufficient to cure the most obstinate cases. Now and then a case will ueed longer treatment. In most cases one packing is sufficient. The uterine canal has remained patulous for months after this treatment in many of my cases, and the tissues of the cervix were soft. The amount of watery oozing while the gauze is in place is very large, but soon decreases after its removal. Local depletion seems to be assured.

'The gauze although left in in some instances as long as ten days has never come out anything but perfectly sweet.

I have made a tabulated statement of twenty-seven cases of chronic endometritis that were treated by curetting and gau\%e drainage; being all the cases, exclusive of Alexander-Adams operation, so treated by me in the six months from February to September of this year. Five of them were fibroids, Nos. 23 to 27 inclusive. In these the relief was only temporary, both as regards symptoms and auatomical results. Of the remaining twenty-two, three, Nos. 4,13 and 20, were unsuitable cases for this treatment. As the result proved, the general symptoms of hysteria far outweighed in importance the local symptoms. One case, No. 12, had an attack of pelvic inflammation following the operation and was made worse by it. This was a hospital case; and I think I can place the fault in asepsis that caused the bad result. In the other eighteen cases the results were very satisfactory. I have not included my Alexander cases, because there the results might fairly be attributed to the restoration of the uterus to a more normal position in the pelvis. There was a rise in temperature on the second or third day in four cases, Nos. 10, 12, 17 and 19. In No. 10 there was a cystitis due to the use of the catheter, and the temperature night well be attributed to that. No. 12 we have just referred to. Nos. 17 and 19 were undonbtedly instances of the rise of temperature being due to defective drainage, removing the gauze in every instance brought the temperature down. In Nos. 5 and 19 I tore into the broad ligament on the left side while divulsing, without any subsequent harm in either case. Five cases, Nos. 2, 7, 10, 17 and 20, had had previous local treatment for periods ranging from nine months to two and a half years without relief. No. 2 had been under treatment by gradual dilatation of the servical canal, douches, etc., for nine months without any improvement in her symptoms; and in spite of the fact that she had both ovaries prolapsed and one of them much enlarged, she was entirely relieved for six inonths, and then suffered only from a dragging sensation, due undoubtedly to the prolapsed ovaries and hard work. No. 7 had had an operation on the cervix and perineum a year before by one of our foremost operators, without relief. In two cases, Nos. 14 and 21 , the curetting was done as a preliminary to abdominal section, with the object of doing away with the diseased condition of the endometrium before removing the more serious trouble in the adnexa. In No. 14 the curetting and drainage had the effect of relieving the pain and diminishing the purulent discharge. (The temperature dropped from $102^{\circ}$ to normal.) In No. 21 it relieved the pain, and the temperature fell from $101.4^{\circ}$ gradually until it reached normal on the third day.

'To sum up the advantages that this method of treatment of chronic endometritis seems to possess, we may enumerate; its qujckness of perforinance ; its thoroughness; the locul depletion of the pelvis, including drainage of the Fallopian tube if their uterine ends are not occluded; and, in cases of stenosis a permanent increased calibre of the uterine canal.

In closing, I wish to say that this method of local treatment, like any other, should be accompanied by hygienic and tonic general treatment to ensure the best results.

\section{FIVE CASES OF APPENDICITIS.'}

ISY ITKNIY W. BROUGITON, M.D., JAMAIUA PLAIN, BOSTON.

TuE following cases present some interesting features, and may add something to the study of an important disease. I make the report from the standpoint of a general practitioner, who sees these cases early, and upon whom devolves considerable responsibility. He must usually make the diagnosis, which is not always easy. Moreover, he must learn to weigh the value of the earlier symptoms; for a correct interpretation of these will be his guide as to the proper time for consultation, and the settling of the important question of operation. Just here, as I understand it, lies a difficult problem. How to differentiate be1 lend before the Boston Society for Medical Observation, December 5,1892 . 